\title{
Editorial
}

Copyright $\odot 2018$ The Author(s). Published by BCS, The Chartered Institute for IT under Creative Commons license http://creativecommons.org/ licenses/by/4.0/

\section{The robot will see you now?}

Piers A. C. Gatenby

Visiting Professor, Department of Clinical and Experimental Medicine, University of Surrey, Guildford, UK

\section{ABSTRACT}

Remote medical videoconferencing and teleconsultations are well-established in modern medical practice with good video and audio quality and data security. Extending this practice to ward rounds requires bringing the communication interface to the ward patient. This was found to be acceptable to patients and technically successful by Croghan et al.

Keywords: robot, ward round, innovation

In the first issue of this volume, Croghan et al. ${ }^{1}$ present their initial experience using a system of a tablet computer mounted on a remotely controlled mobile chassis to allow a consultant surgeon to conduct a remote ward round in the presence of the other members of the surgical team. The system is inexpensive, requires minimal installation and utilise existing hospital infrastructure. The study shows initial proof of concept. In the context of the general surgical ward rounds at a single centre in Ireland, the robotic ward rounds were acceptable to a range of patients of different ages, a wide variety of pathologies and the robotic ward rounds were generally successfully completed.

The concept of telemedicine/remote consultations is not new, particularly for outpatient consultations and inter-professional teleconferencing, but as the authors state, there has not been wide uptake of this technology for inpatient ward rounds. Teleconferencing interfaces are well developed with data security, good video and audio quality, but the interfaces have not generally been designed to be independently mobile.

Some general surgical inpatients are relatively immobile (due to frailty, comorbidity, the presence of tubing, drains, monitoring leads, etc.) and despite a cultural change to earlier mobilisation, the most workable solution is to bring the surgical ward round to the patient rather than vice versa, whereas for other applications of telemedicine, portability of the communication interface has not been necessary. This has been the major technical impediment to allowing ward rounds to be conducted remotely. With the system used by Croghan et al., the only reported technical difficulty was secondary to the wireless data connection (which was resolved by an upgrade to the bandwidth). Successful telerounding using more expensive robots was previously reported for urology inpatients in $2007^{2}$ and in general surgery by Imperial College in $2005 .^{3}$ Uptake since that time has been poor, despite the positive descriptions of safety, staff and patient acceptability and technical success. There has also been limited information published on the time taken for the robotic ward rounds compared to conventional rounds.

There have been two developments within the last decade, which will help with wider adoption of this technology. The first of these is the widespread use of good quality, affordable videoconferencing (in particular hand-held tablets) used not just in business but also at home. Patients are now frequently familiar with these 
types of systems prior to their hospital admissions, when unanticipated initial use of new communication technology whilst being unwell would be an unacceptable step for many patients. Younger patients, those who are most proficient with computers and those who have previously used video calling facilities were reported to have the greatest acceptance of teleconsultation in primary care. Deprivation was associated with lower chance of previous use of video calling. ${ }^{2}$ The second is the functioning system to move the communication interface around the ward round. Croghan et al. have mounted their commercially available tablet onto an inexpensive lightweight Double ${ }^{\mathrm{TM}}$ base mobility unit. The ward round is conducted with the other members of the surgical team, who will be in a position to guide and assist the robot, for example, through doorways, over steps, into (and operating) lifts and avoiding other obstacles. To this end, the transportation for the tablet is lightweight but stable and manoeuvrable. A simple alternative to this would be for the surgical team to carry the tablet computer and hold it/pass it to the patients for the consultation. In many hospitals, individual bedside screens have been installed providing television, telephone and internet services, which, if they include cameras, etc., could potentially be used for these teleconsultations. The drawbacks to removing the base mobility unit are the loss of image stability and ability of the remote consultant to direct the camera (as well as the need to have a mechanism to allow cleaning of the device and prevention of cross infection).

The robotic ward rounds have still required the presence of the other members of the surgical team and, with the need for physical examination and practical procedures on the ward, cannot eliminate the need for this physical surgical presence. The remote consultant will not be able to pick up on nonvisual/audio information such as touch, smell and temperature and there have been limitations with other interactions and cues; however, many of the shortcomings in interactions may be overcome with time ${ }^{4}$ and reduced by technological advances.

The addition of further software to this system such as pathology results, radiology images and observation charts (which are commonly available at the bedside on

\section{REFERENCES}

1. Croghan SM, Carroll P, Ridgway PF, Gillis AE and Reade S. Robot assisted surgical ward rounds: virtually always there. Journal of Innovation in Health Informatics 2018;25(1):41-56.

2. Ellison LM, Nguyen M, Fabrizio MD, Soh A, Permpongkosol $S$ and Kavoussi LR. Postoperative robotic telerounding: a multicenter randomized assessment of patient outcomes and satisfaction. Archives of Surgery 2007;142(12):1177-81. Available from: https://doi.org/10.1001/archsurg.142.12.1177. PMid:18086984.

3. Imperial College London. "Sister Mary" the robo-doc to start making ward rounds at St Mary's Hospital [Internet]. Available from: https://www.imperial.ac.uk/college.asp?P=6377. Accessed 8 October 2017. conventional ward rounds) could further increase the quality of remote ward rounds and improve care. These systems could be enhanced by employing computer-aided decision making with these data, ${ }^{5}$ but it is important to ensure that proper governance with regard to data security and access is maintained. ${ }^{6}$

The most likely early adopters of robotic ward round systems would be remote locations where consultants will visit but are not present for patients' entire hospital stay, a specialist opinion (not present on site) is required or when surgeons have to work at another site or are at a conference.

The robotic ward rounds have not been designed to replace all conventional ward rounds and in-person consultations are very important to build the physician-patient relationship. ${ }^{7}$ Some aspects of care can be undertaken by other members of the surgical team (including physical examination and practical procedures) and standardised care pathways also assist in maintaining quality of care without direct consultant presence. Specialist physical/operative procedures (with the exception of those carried out by robotic surgery) require expert senior surgical presence. Croghan et al. and Ellison et $a .^{2}$ both reported patient satisfaction with the robotic ward rounds or a preference for a teleround by their own surgeon rather than a colleague in person, but not all patients will accept or are suitable to have their consultations via telemedicine. In the majority of hospitals, many ward rounds are undertaken by other members of the surgical team without the consultant. The surgical team could then discuss the patients' management with the consultant if needed via telephone or away from the ward with the option of viewing/communicating other clinical information remotely. This well-established and effective system is ingrained in surgical practice and robotic ward rounds would need to offer significant advantage for wider adoption.

In summary, it is likely that the current high-quality video/ audio and low system costs will allow for wider adoption of mobile telerounding technology than the previous robotic ward round systems, but whether there is a wide-enough need for fully autonomously mobile teleround systems over the alternatives such as a tablet alone or telephone calls to the other members of the surgical team has yet to be seen.

4. Morrison C, Fitzpatrick $G$ and Blackwell A. Multi-disciplinary collaboration during ward rounds: Embodied aspects of electronic medical record usage. International Journal of Medical Informatics 2011;80(8):e96-111. Available from: https://doi. org/10.1016/j.ijmedinf.2011.01.007. PMid:21334969.

5. de Lusignan S. In this issue: time to replace doctors' judgement with computers [Internet]. Journal of Innovation in Health Informatics 2015;22(3). Available from: https://hijournal.bcs.org/ index.php/jhi/article/view/839. Accessed 8 October 2017.

6. de Lusignan S, Liyanage $\mathrm{H}$, lorio CTD, Chan T and Liaw S-T. Using routinely collected health data for surveillance, quality improvement and research: framework and key questions to 
assess ethics, privacy and data access. Journal of Innovation in Health Informatics 2016;22(4):426-32. Available from: https:// doi.org/10.14236/jhi.v22i4.845. PMid:26855276.

7. Kirthi V, Ingham J, Lecko C, Amin Y, Temple RM, Hughes S, et al. Ward rounds in medicine: principles for best practice
[Internet]. London, UK: RCP, 2015. Available from: https://www. rcplondon.ac.uk/projects/outputs/ward-rounds-medicine-principles-best-practice. Accessed 8 October 2017. 